\title{
Clogging Report with Ad-Diffusion and Carter Performance Inspection using Intelligent Vanet
}

\author{
Aswiga R.V. \\ Angel College of Engg. \\ and Technology \\ Tirupur, TamilNadu, \\ India

Praveena R.
Angel College of Engg. and
Techology
Tirupur, TamilNadu,
India

\author{
Rajeswari M. \\ Dept Of CSE \\ Angel College of Engg \\ and Technology \\ Tirupur, TamilNadu, \\ India
}

\author{
Umamaheswari P. \\ Dept Of CSE \\ Info Institute Of \\ Technology \\ TamilNadu, \\ India
}

Jayanthi S.

Angel College of Engg and

Technology

Tirupur, TamilNadu, India

\begin{abstract}
Mobile Ad-hoc network is a collection of mobile nodes that form a temporary network without use of any existing infrastructure. Group communication is challenging task in MANET .In recent years many protocols have been developed to achieve group communication in MANET. Since VANET is the subset of MANET the same group communication would be achieved to improve the scalability, reliability and optimality of routing protocols. Using group communication, distributed traffic information system has evolved for detecting traffic problems on road. The traffic information will be communicated to vehicles in the form of V2V or V2I interaction medium. In the existing system, the traffic information with the vehicle id, position, location along with the ad dissemination, driver behavior information will be transmitted to other vehicle which leads to unnecessary tracking of vehicles on the road side. This in case leads to major threats such as attacks, hacking vehicles, etc...Therefore in proposed system the security in VANET is obtained by restricting the unnecessary flow of information like vehicle id or position to other ongoing vehicles on the roadside with the help of trust based reputation system in VANET. The ad dissemination along with traffic information and driver behavior information will be transmitted to other vehicles in the existing system with the help of trust based reputation system in VANET. But since it contains some disadvantages, the fuzzy logic reputation system will be implemented as our future work where the architecture is decentralized.
\end{abstract}

Keywords-CEPA (Complex Eventual Parallel Architecture), CIS (Congestion Information System), IS (Information System), Agents, Vanet parallel ad dissemination architecture 


\section{INTRODUCTION}

With the rapid growth of traffic in internet, congestion control has become one of the most important issues in recent communication networks and in modern transmission technologies.But during the last decade's global road traffic has been increasing day by day. This leads to the fact that currently, road traffic congestion are one of the most common criteria that motorists have to tolerate in their trips. Changqiao Et al [4] illustrates that one of the most important topic in intelligent transportation system is the development of replicated congestion information systems (CIS).This system will identify the blockage conditions on the road and try to detect and avoid traffic flow difficulties through sequential delivery of messages between vehicles. Replicated congestion information system (CIS) will avoid the constraints on the road side and try to prevent the difficulties on the road side equipment approaches. Communication between vehicles is usually achieved with the help of ad-hoc network. So VANET is a movable dynamic network in which the nodes are designated as vehicles that travel on a Road and communicate with each other through wireless technology. Each vehicle in the network will broadcast data messages called beacons, which contains its current velocity, speed, position, etc...In this manner each vehicle of VANET can identify the neighboring vehicles that travel along the roadside. However these broadcasted messages imply a large number of events across the VANET that each vehicle must deal in the network. To cope with large number of events like accidents, traffic jam and other natural disasters, an eventual architecture has been developed as a new architectural paradigm. Eventual architecture is a software model which processes the events with low delay where the events should be processed as soon as they arrived. Currently eventual architecture plays an important role in many e-commerce areas such as financing or goods distribution. Furthermore this is expected to grow in future as long information systems need to evaluate data from many more replicated sources. In an E-commerce domain a certain information systems are used and frequent real world incidents on the road are designated as events in first layer of information systems (IS).These incidents have complex relationships that contain similar patterns. Real time incidents here indicate accidents, traffic jam and other natural disasters that occur on the road. Complex relationship deals with vehicles that are travelling below the average speed on the road and group these vehicles into a cluster. Because of processing these real time incidents in the eventual architecture, it is possible to become aware of target real world activity by checking the information systems and looking for these similar patterns. Consequently the key task is the subsequent identification of particular event pattern from the stream of events that flow through IS. This is the sophisticated form of software architecture that deals with numerous heterogeneous events from different streams. This type of system is suitable and adoptable for replicated environments where dispersed elements can act as data a source which provides useful information about the environment in which vehicles travel along the road side. In this paper we proposed an architecture in such a way that accepts the data's from different sources but process only beacon messages in order to detect the traffic or blockage conditions on the road. Other messages will not be processed inside the 
architecture except the beacon messages. The input for beacon messages from different sources web sources, on-board sensors, vehicles, etc. This method helps to identify the traffic conditions and differentiate it based on different lanes regardless of the communication protocol used. This takes real world map thus covering large amount of data's from road side, This paper takes an initial approach to filter only the traffic messages along with ad disseminations and identify whether the driver is alive or not and process the information containing the corrective action to other vehicles .Fernando et al [1] demonstrated that the security is enhanced by restricting the flow of information like vehicle id, location, etc to other vehicles in order to avoid major threats like terrorist attack, robbery, etc..Parallel traffic management system will be implemented as future work.

\section{RELATED WORK}

Vehicular ad-hoc network has widely been used to develop traffic information system (TIS). This traffic system gathers information from messages to check the traffic conditions on the road. This reduces the accidents by alerting motorists who is travelling on the congested location. Based on this we propose a system called as a replicated congested information system (CIS).In this system each vehicle communicates only the traffic messages to other vehicles in a secure way. In this approach we extend the architecture as parallel architecture that guarantees the following different characteristics like choosing the alternative path to reach the destination and control the traffic with the help of security measures for various transportation services which includes public vehicles, taxies, metro, fire trucks, ambulances, etc...Fluctuation of traffic status is very difficult in order to predict and control the traffic messages. In addition to the existing work we also included the ad dissemination along with the driver's behavior detection system that transmits the corrective action to the current vehicle and also to other ongoing vehicles on the road side. Thus in this paper we reproduced the architecture in such a way that it allows dissemination of traffic information along with the ad dissemination and produces safety measures that guides other vehicle driver's to take corrective action from the existing information provided.

\section{ARCHITECTURE OVERVIEW}

EDA act as a middleware between network layer and back-end application. But we are adding an additional parallel system next to EDA which acts parallel and provide decisions to be made in case of any accidents or some natural disasters on the roadside. This is called as CEPA (Complex Eventual Parallel Architecture). This architecture will act parallel by taking into account the input from different sources like EDA, weather conditions, external source agent, etc.., On the other hand, network layer handles the dissemination and reception of traffic messages throughout the VANET. Our proposed architecture also provides an interface that allows external entities to access information from underlying VANET in a quite efficient way. This CEPA generates a traffic alarm whenever a traffic jam is detected. This type of event is sent to the back end application. So that they could use this information to alert both the driver and the information panels of the motorway 
depending on where CEPA is running. In Saif Al-sultan et al [3] the driver's behavior is detected with the help of sensors which is situated inside the vehicles. This will be monitored on the parallel system in order to take decision and send the message to other ongoing vehicles on the roadside to prevent accidents from happening. This parallel architecture will detect the abnormal behaviors which is exhibited by drivers and based on that evaluate the driver's condition while travelling on the road.CEPA generates different agents like traffic filter agent (TFA) to filter the traffic related information and identify the slow vehicles which is travelling on the road with the help of SVA (slow vehicle agent).SVA will detect the vehicles which are running below the average speed or even stopped in a busy environment. The vehicles will usually slow or stop flowing because of traffic or blockage which can be identified by SVA.Warning agent (WA) will provide warning messages to other vehicles after classifying the traffic level such as slight, moderate and severe. In addition to the above agents, the adaptation agent (AA) and external source agent (ESA) helps to take intelligent decisions. In previous work [20] we did not identify the decrease in diffusion rate of information when it reaches the far vehicles. But here we identify the diffusion rate and overcome this problem by designating each RSU to be a SRSU (source road side units). That is when the message needs to be delivered to the far vehicles and when the destination is in large distance; the intermediate relays can act as source hence the transmission is done with the help of logical transmission via signals. Since the signal is reached from the source which is nearer to the destination vehicle, the message will be delivered to the vehicle as designated by the sender. Here the malicious nodes will not transmit wrong information to vehicles because trust based reputation system is used here. The figure one demonstrates the work flow process of vanet reputation system architecture.

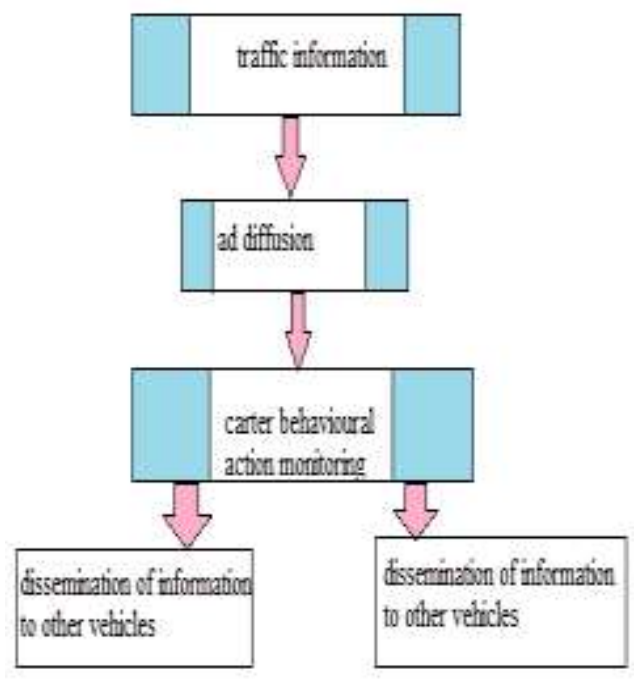

Fig.1. Work Flow Process Diagram

In the Fig. 3 shown below demonstrates that, from the network layer different messages will be transmitted to the complex EDA. But this architecture will filter only the traffic messages with the help of TFA and identify the slow moving vehicles that are below the average speed .After getting input from various external source agent, if the blockage is detected then the adaptation agent will take corrective action with the help of experts knowledge and pass this information to parallel architecture which evaluates drivers behavior and calculate the number of vehicles flow rate for that particular location to help the vehicle to reach the destination in short time. Finally warning agent will send the 
alarm to other ongoing vehicles on the road by restricting the traffic id in order to avoid security threats. As shown in this figure below control unit 1 passes only ad dissemination details. Control unit 2 passes driver behavior detection system information to other ongoing vehicles on the road side. Control unit 3 passes only warning message regarding the traffic information to other ongoing vehicles on the road side.

\section{AD-DIFFUSION RATE}

An SP refers to a business entity with a fixed position, such as a restaurant or a gas station. Each node periodically broadcasts beacons containing the driving states, such as location, speed, and heading direction, to support traffic safety applications. First, each SP intends to maximize its Advertising effect by disseminating ads to as many nodes as possible. Second, as an ad receiver, each node would like to learn of the local services without being distracted by excessive ads. In addition, being selfish, each node forwarding one ad expects to receive certain incentive in return. Third, VANETs as a whole need to ensure ad dissemination is under control in the face of increasingly more ads to avoid message storms. Since the information is delivered in the logical manner from the relay which acts as a source, the quality of message is high. The quality here is considered to be reliable. Reliable in the sense the message is not affected by malicious nodes or the attackers cannot change the content of message. Here the first vehicle transfers the information to other vehicles which act as a source unit that contains Fuzzy reputation system in vanet. The red color nodes obtain information from other vehicles but it act as a source when it delivers the message to other vehicles. The node stops acting as a source when it designates to be a final node.

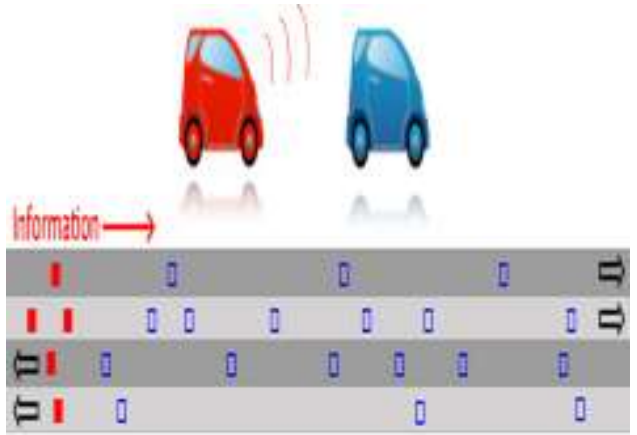

Fig.2. Clogging Report with Ad-Diffusion

\section{VANET PARALLEL REPUTATION SYSTEM ARCHITECTURE IMPLEMENTATION}

$\mathrm{AD}$ is proposed to support secure ad dissemination with pragmatic cost and effect control. To trade off the conflicting requirements of the involved parties, an incentive-centered architecture is proposed for VAAD, where the SP pays other entities for their services in ad dissemination. Constrained by the incurred cost, each SP will set a realistic advertising effect requirement in terms of the number of ad receivers and the ad rebroadcast frequency. The VAAD Manager (VM) is introduced to coordinate the interactions between SPs and VANETs. Upon receiving a dissemination request from one SP with cost and effect specifications, the VM will obtain proper authorization from VANET Authority for this ad. With the authorization, the VM can request one SRSU to disseminate the ad according to the specifications. The goal of trust management in VANETs is not limited 
to reliable package delivery. One main aim of VANETs is to increase road safety and reduce traffic congestion by allowing information sharing among peers about road and traffic conditions. Trust management in VANETs should help peers detect false information provided by malicious nodes and make informed driving decisions. Trust management in this case is more challenging than that for reliable package delivery. Much dynamics has to be taken into consideration, such as the time and location of reported events, and the types of the events. Thus, previous trust modeling endeavors in mobile Adhoc networks become worthless when being directly applied

to vehicular ad-hoc networks. Trust management can effectively improve peer collaboration in VANETs to share information and detect malicious peers. However, the trust management itself may become the target of attacks and be compromised. Thus fuzzy logic based reputation system will be considered as our future work.

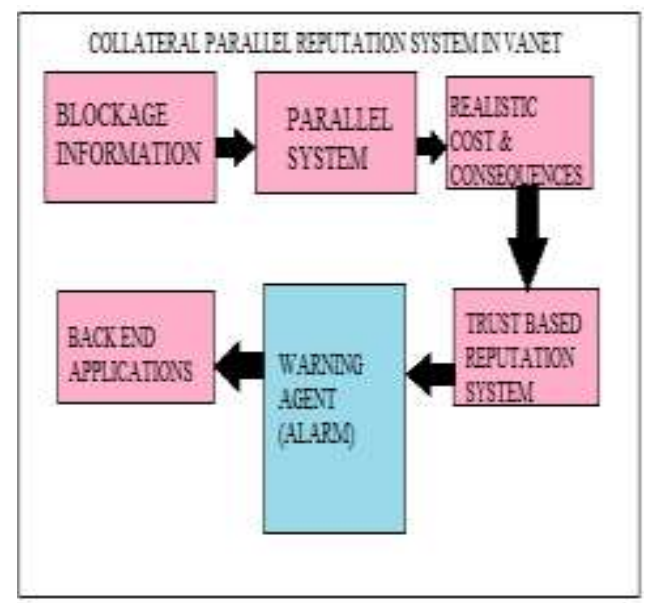

Fig.3.Vanet Parallel Reputation system Architecture implementation

\section{SIMULAITON STUDY}

\subsection{Performance Evaluation}

The simulation results bring out some important characteristic function of Region based clustering mechanism. In this section we record the Cost and Overhead Comparisons and packet ratio parameters of the simulation by using record procedure. The parameters includes Packet delivery ratio, Throughput, Packet loss rate, delay time etc. The recorded events are stored in the trace files. The number nodes is going to be participated in the simulation is decided. As it is a VANET environment, the topology changes dynamically. Here we are using only a logical topology as it is wireless environment. By executing the trace files by using xgraph or gnuplot we can get the graph as the output.

\subsection{Simulation Parameters}

With regard to the evaluation of the CEPA, the following two different measurements have been used: 1) the Evaluated/Detected rate (DR) and 2) the mean time to detection (MTTD). Both Measurements are defined by the following equations:

Detected Rate $=$ Total No of detected traffic

Number of traffic jams On the Lane

$M T T D=1 n \_n I=1($ tdetection - tstart $)$ 


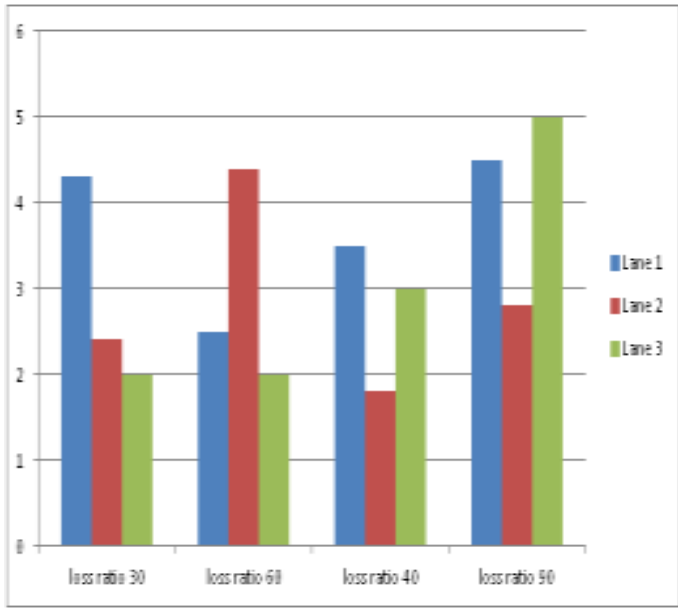

Fig. 4. Estimation rate of the CEPA for the two types of simulated congestions, given different penetration at $\mathrm{y}$-axis and packet-loss rates at $\mathrm{x}$-axis.

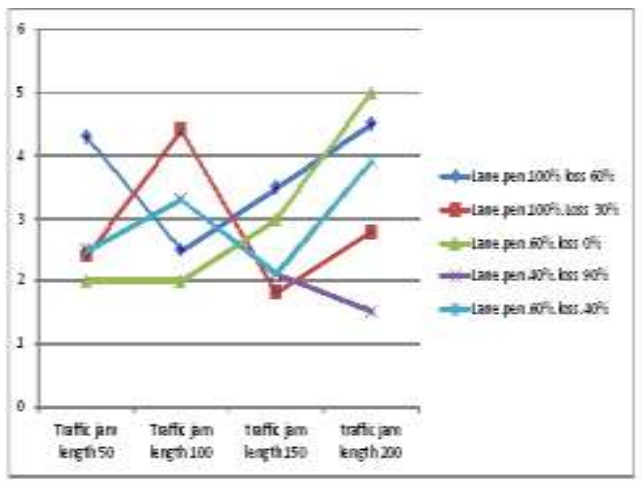

Fig. 5. MTTD of the lane and raw modes, given some penetration rates $(60 \%, 40 \%, 100 \%)$ and loss ratios $(60 \%, 30 \%, 40 \%, 100 \%)$ for a three-lane-type traffic jam.

\section{CONCLUSION}

In this paper, a complex eventual parallel architecture along with Ad dissemination has been put forward as a method for identifying traffic congestions in the context of a www.ijcat.com replicated CIS. In particular, this architecture may run as part of onboard equipment in a vehicle or in an external module of the road infrastructure. The system processes the beacon messages, and it can detect different levels of congestion on a road; moreover, it can add environmental information such as the current weather condition plus the behavior of the driver so that the traffic jam detection is enriched. Results from the different simulations state that this architecture can detect several types of traffic jams. Thus, the vanet parallel ad dissemination architecture achieved better results, as long as the traffic jam covered several lanes. Therefore in proposed system the security in VANET is obtained by restricting the unnecessary flow of information like vehicle id or position to other ongoing vehicles on the roadside. Tests also put forward that the penetration rate of the message does not affects its performance and reliability. As explained throughout the test section, this case is because of the way the traffic density is calculated, which directly relies on the number of slow vehicles detected.

In this paper, the following main further research topics have come up 1)the first topic will focus on modifying the vanet parallel ad dissemination architecture to use virtual segments or clustering techniques instead of the segments provided by a digital map to divide the road during the CEP processing. This way, the problems that might arise when the road segments are long could be sorted out. 2) FUZZY LOGIC based reputation system will be enhanced to punish the malicious nodes

\section{REFERENCES}


International Journal of Computer Applications Technology and Research

Volume 2- Issue 5, 628 - 636, 2013

[1] Fernando Terroso, Rafael, "A Cooperative Approach To Traffic Congestion Detection With Complex Event Processing And Vanet,"in IEEE Transaction On Intelligent Transportation Systems, Vol 13,NO 2,JUNE 2012.

[2] Changqiao ,Hongke,’QoE-Driven User Centric VoD Services Multihomed P2P-Based Vehicular Networks",in IEEE Transactions on vehicular technology,vol 62,no 5,JUNE 2013

[3] Saif, Ali,"Context Aware Driver Behavior Detection System in Intelligent Transportation Systems (ITS)", in IEEE Transaction On Vehicular Technology,

[4]Ramin,"DTN Routing Protocols for VANETs:Issues and Approaches", in IJCSI ,Vol 8,Issue 6,No 1,November 2011

[5] E. Strom, H. Hartenstein, P. Santa, and W. Wiesbeck, "Vehicular communications: Ubiquitous networks for sustainable mobility," Proc. IEEE,vol. 98, no. 7, pp. 1111-1112, Jul. 2010.

[6] D. Luckham, The Power of Events: An Introduction to Complex Event Processing in Distributed Enterprise Systems. Reading, MA: Addison- Wesley, 2002.

[7] O. Etzion, "Towards an event-driven architecture: An infrastructure for event processing position paper," in nRules and Rule Markup Languages for the Semantic $W e b$. Berlin, Germany: Springer-Verlag, 2005, pp. 1-7.

[8] M. P. Gardner, "Highway traffic monitoring," Committee on Highway Traffic Monitoring, Washington, DC, Tech. Rep., 2000.
[9] H.-Y. Cheng and S.-H. Hsu, "Intelligent highway traffic surveillance with selfdiagnosis abilities," IEEE Trans. Intell. Transp. Syst., vol. 12, no. 4, pp. 1462-1472, Dec. 2011.

[10] R. Wang, L. Zhang, R. Sun, J. Gong, and L. Cui, "Easitia: A pervasive traffic information acquisition system based on wireless sensor networks,"IEEE Trans. Intell. Transp. Syst., vol. 12, no. 2, pp. 615-621, Jun. 2011.

[11] S. Vaqar and O. Basir, "Traffic pattern detection in a partially deployed vehicular ad hoc network of vehicles," IEEE Trans. Wireless Commun.,vol. 16, no. 6, pp. 40-46, Dec. 2009.

[12] R. Bauza, J. Gozalvez, and J. SanchezSoriano, "Road traffic congestion detection through cooperative vehicle-to-vehicle communications," in Proc. 4th IEEE LCN Workshop User Mob. Veh. Netw., 2010, pp. 606-612.

[13] D. F. Llorca, M. A. Sotelo, S. Sánchez, M. Ocaña, J. M. Rodríguez- Ascariz, and M. A. García-Garrido, "Traffic data collection for floating car data enhancement in V2I networks," EURASIP J. Adv. Signal Process.,

vol. 2010, pp. 5:1-5:13, Mar. 2010.

[14] I. Leontiadis, G. Marfia, D. Mack, G. Pau, C. Mascolo, and M. Gerla, "On the effectiveness of an opportunistic traffic management system for

vehicular networks," IEEE Trans. Intell. Transp. Syst., vol. 12, no. 4, pp. 1537-1548, Dec. 2011. 
International Journal of Computer Applications Technology and Research Volume 2- Issue 5, 628 - 636, 2013

[15] A. Skordylis and N. Trigoni, "Efficient data propagation in trafficmonitoring vehicular networks," IEEE Trans. Intell. Transp. Syst., vol. 12, no. 3, pp. 680-694, Sep. 2011.

[16] M. Saito, J. Tsukamoto, T. Umedu, and T. Higashino, "Design and evaluation of intervehicle dissemination protocol for propagation of proceeding traffic information," IEEE Trans. Intell. Transp. Syst., vol. 8, no. 3,pp. 379-390, Sep. 2007.

[17] A. Adi, D. Botzer, G. Nechushtai, and G. Sharon, "Complex event processing for financial services," in Proc. IEEE Serv. Comput. Workshops, 2006, pp. 7-12.

[18] N. Museux, J. Mattioli, C. Laudy, and H. Soubaras, "Complex event processing approach for strategic intelligence," in Proc. 9th Int. Conf. Inf. Fusion, 2006, pp. 1-8.

[19] J. Dunkel, A. Fernández, R. Ortiz, and S. Ossowski, "Event-driven architecture for decision support in traffic management systems," Expert Syst. App., vol. 38, no. 6, pp. 6530-6539, Jun. 2011.

[20] R.V.Aswiga, R.Praveena, S.jayanthi, R.Maheswari," Parallel Ad-Dissemination With Congestion Information And Driver Behavior Monitoring In Intelligent Vehicular Networks,'IJESIT, vol 2 ,issue 5 ,sept 2013. 